\title{
FRAGMENTATION AND EVOLUTION OF MOLECULAR CLOUDS. III. THE EFFECT OF DUST AND GAS ENERGETICS
}

\author{
Hugo Martel ${ }^{1,2}$, Andrea Urban ${ }^{3}$, and Neal J. Evans I $^{4}$ \\ ${ }^{1}$ Département de Physique, de Génie Physique et d'Optique, Université Laval, Québec, QC, G1V 0A6, Canada \\ ${ }^{2}$ Centre de Recherche en Astrophysique du Québec, C.P. 6128, Succ. Centre-Ville, Montréal, QC, Canada \\ ${ }^{3}$ Sapling Learning, Inc., 2815 Exposition Blvd, Austin, TX 78703, USA \\ ${ }^{4}$ Department of Astronomy, University of Texas, 2515 Speedway, Stop C1400, Austin, TX 78712-1205, USA \\ Received 2012 May 29; accepted 2012 July 29; published 2012 September 5
}

\begin{abstract}
Dust and gas energetics are incorporated into a cluster-scale simulation of star formation in order to study the effect of heating and cooling on the star formation process. We build on our previous work by calculating separately the dust and gas temperatures. The dust temperature is set by radiative equilibrium between heating by embedded stars and radiation from dust. The gas temperature is determined using an energy-rate balance algorithm which includes molecular cooling, dust-gas collisional energy transfer, and cosmic-ray ionization. The fragmentation proceeds roughly similarly to simulations in which the gas temperature is set to the dust temperature, but there are differences. The structure of regions around sink particles has properties similar to those of Class 0 objects, but the infall speeds and mass accretion rates are, on average, higher than those seen for regions forming only low-mass stars. The gas and dust temperature have complex distributions not well modeled by approximations that ignore the detailed thermal physics. There is no simple relationship between density and kinetic temperature. In particular, high-density regions have a large range of temperatures, determined by their location relative to heating sources. The total luminosity underestimates the star formation rate at these early stages, before ionizing sources are included, by an order of magnitude. As predicted in our previous work, a larger number of intermediate-mass objects form when improved thermal physics is included, but the resulting initial mass function (IMF) still has too few low-mass stars. However, if we consider recent evidence on core-to-star efficiencies, the match to the IMF is improved.
\end{abstract}

Key words: hydrodynamics - ISM: clouds - methods: numerical - stars: formation

\section{INTRODUCTION}

The physics of star formation is the link between the smallscale-planet formation-and the large-scale-galactic evolution. Many, probably most, stars form in highly clustered environments (Lada \& Lada 2003; Bressert et al. 2010). Within the highly complex structure of a molecular cloud, theorists have identified the clump as the object that forms a cluster (Williams et al. 2000; McKee \& Ostriker 2007). Observational studies have found a range of structures that might correspond to this concept, but the formation of rich clusters seems most clearly associated with particularly dense clumps, identified by strong emission from tracers of dense gas (e.g., Wu et al. 2010).

This paper is the third of a series that studies the fragmentation of a dense molecular clump using smoothed particle hydrodynamics (SPH) to follow the hydrodynamics, with a focus on the effects of the thermal physics. In Paper I (Martel et al. 2006, hereafter MES06), we showed that an isothermal gas will fragment excessively, producing only very low mass stars. In Paper II (Urban et al. 2010, hereafter UME10), we included global radiative feedback from the forming stars, assuming that the gas temperature was equal to the dust temperature; this approximation overproduced high-mass stars. In this paper, we calculate the gas temperature separately from the dust temperature. We use these simulations to address the role of thermal physics in the fragmentation problem, the density distribution around forming stars in a proto-cluster, the evolution of the farinfrared luminosity during formation, with application to the use of far-infrared luminosity as a probe of star formation rate, and the effects of an improved thermal physics on the mass function of forming stars.
In our previous work (UME10) we modeled a clustered starforming region and showed that dust-gas thermal energetics with source luminosity terms from young stars can heat the gas and prevent fragmentation. Less fragmentation led to the formation of massive stars, which had not been produced in our isothermal simulation (MES06). Although we were able to form massive stars, we missed a significant fraction of the low-mass stellar population. We hypothesized in UME10 that by including a more realistic dust-gas thermal energetics algorithm we would increase the number of low-mass stars. Including molecular cooling would decrease the temperature of the gas, leading to more fragmentation and more lowmass stars. To test our hypothesis, we have implemented the complete heating and cooling algorithm described in Urban et al. (2009, hereafter UED09) in simulations with similar scales and parameters as the simulations discussed in UME10. We discuss our work in the following sections. In Section 2, we discuss our numerical algorithm and our new method of calculating the gas temperature. In Section 3, we discuss the initial conditions and parameters of our simulation. Our results are discussed in Section 4. We conclude and summarize the paper in Section 5.

\section{THE NUMERICAL ALGORITHM}

Our numerical algorithm was described in MES06 and UME10. It is a standard SPH algorithm (see Monaghan 1992, and references therein), which simulates the growth of structures in a cubic volume with periodic boundary conditions, representing a small part of a giant molecular cloud. The code was modified to include particle splitting and sink particles. In the optically thin regime, the Jeans mass decreases with increasing 
Table 1

Numerical Parameters of the Simulations

\begin{tabular}{lccccccc}
\hline \hline Run & $\begin{array}{c}T_{\min } \\
(\mathrm{K})\end{array}$ & $T_{\mathrm{K}}$ & $\begin{array}{c}M_{\text {tot }} \\
\left(M_{\odot}\right)\end{array}$ & $\begin{array}{c}L_{\text {box }} \\
(\mathrm{pc})\end{array}$ & $\begin{array}{c}M_{J}^{\text {init }} \\
\left(M_{\odot}\right)\end{array}$ & $\begin{array}{c}M_{J} \\
\left(M_{\odot}\right)\end{array}$ & $\begin{array}{c}r_{\text {acc }} \\
(\mathrm{AU})\end{array}$ \\
\hline I05 & 5 & $T_{\text {min }}$ & 671.4 & 0.984 & 0.617 & 0.0080 & 152 \\
D05 & 5 & $T_{\text {dust }}$ & 671.4 & 0.984 & 0.617 & 0.0080 & 152 \\
G05 & 5 & $T_{\text {gas }}$ & 671.4 & 0.984 & 0.617 & 0.0080 & 152 \\
G10 & 10 & $T_{\text {gas }}$ & 1898.0 & 1.390 & 1.744 & 0.0226 & 215 \\
\hline
\end{tabular}

density. Eventually, when the density becomes sufficiently high, the gas becomes optically thick and the Jeans mass starts increasing with density. Hence, there is a minimum Jeans mass, corresponding to the transition between the two regimes. To properly follow the fragmentation of the cloud, it is essential to resolve that minimum Jeans mass. Particle splitting (see Kitsionas \& Whitworth 2002; MES06) enables us to do this at a reasonable computational cost.

When the gas reaches a certain critical density $\rho_{c}$, the algorithm replaces gas fragments by sink particles, using the method of Bromm et al. (2002). Sink particles (or sinks) represent protostellar cores. They are not allowed to fragment or merge, but they have the ability to grow by accreting surrounding gas particles. Any bound gas particle within its accretion radius, $r_{\text {acc }}(\sim 150 \mathrm{AU}$ for all but one simulation, see Table 1), is automatically accreted into the sink. Because $r_{\text {acc }}$ is considerably larger than the actual forming star (likely radius a few to tens of solar radii), the evolution of material inside the sink is unknown. For the simulations, we assume that all the material falling into the sink flows continuously onto the actual stellar core, producing accretion luminosity. The luminosity resulting from that accretion will heat the surrounding gas. In order to calculate the luminosity from a sink particle, we use the models of Wuchterl \& Tscharnuter (2003), specifically their Table 3, which include the effect of mass accretion on the luminosity. For objects with masses greater than $2 M_{\odot}$, we use the method described in UME10 to calculate the luminosity. There is considerable evidence that not all material passing through a radius of $150 \mathrm{AU}$ winds up in the forming star, so we also consider the effects of the loss of some material in comparing to the mass function (Section 4.5).

\subsection{Dust and Gas Temperature Calculation}

We use the same numerical methods described in UME10 to calculate the mass accretion rate onto sinks and the dust temperature. In order to calculate the gas temperature, we use the method of UED09. We give a brief description here.

The dust temperature is determined using the method discussed in UME10, and in more detail in UED09. UED09 used a spherically symmetric radiative transfer code, DUSTY (Nenkova et al. 2000), to calculate the dust temperature distribution around young stellar objects. Using DUSTY, we created a grid of models with input values of luminosity and density distribution. In the simulations presented in this paper, we calculate the luminosity of individual objects based on mass and mass accretion rate using the models of Wuchterl \& Tscharnuter (2003). We also determine the density profile around each of the sinks formed in our simulation. We then use the grid calculated in UED09 to find an analytic fit to the dust temperature distribution around each of the individual sinks. We approximate the dust/gas distribution around sink particles as spherical.

We calculate the density profile around individual sink particles, as in UME10, using spherical shells. We parameterize the density profile with $n_{o}$ and $\alpha$, as the following:

$$
n(r)=n_{o}\left(\frac{r}{1000 \mathrm{AU}}\right)^{-\alpha} \mathrm{cm}^{-3} \text {. }
$$

The symbol $n$ represents the number density of all particles $\left(n=n_{\mathrm{H}_{2}}+n_{\mathrm{He}}\right)$. We assume a ratio $n_{\mathrm{H}_{2}} / n_{\mathrm{He}}=5$, which corresponds to a mean molecular weight $\mu=2.33$. The gas density is $\rho=\mu m_{\mathrm{H}} n$.

Our approximations are complementary to those of most other simulations that include some thermal physics. We do not include compressional heating during collapse, as does Bate (2009). We are not doing radiative transfer during the SPH calculation, but instead using a pre-computed grid as did Smith et al. (2009), and we are assuming spherical distributions of material around sinks. Thus, we will miss some of the effects of geometry included in papers that do not assume spherical symmetry (e.g., Krumholz et al. 2007, 2010; Bate 2009; Offner et al. 2009). On the other hand, we used realistic grain opacities as a function of wavelength (i.e., OH5 dust opacities, Ossenkopf \& Henning 1994, as described in Young \& Evans 2005) in radiative transfer calculations that include non-isotropic scattering and apply to all relevant optical depths, in contrast to mean opacities and other approximations used by most other simulations (e.g., Krumholz et al. 2007, 2012).

The gas temperature algorithm (UED09) includes energy transfer between gas and dust via collisions, gas heating by cosmic rays, and molecular cooling. Heating by photoelectric emission from dust grains is not included because the external ultraviolet radiation is strongly attenuated and the clump we are simulating is assumed to reside deep in a larger molecular cloud. We are not including ultraviolet radiation from the forming stars. The dust-to-gas ratio is taken to be $4.86 \times 10^{-3}$ (Hollenbach $\&$ McKee 1989), and the grain cross section per baryon is $6.09 \times 10^{-22} \mathrm{~cm}^{2}$ (Young et al. 2004). The cosmic-ray ionization rate is $3.0 \times 10^{-17} \mathrm{~s}^{-1}$ (van der Tak \& van Dishoeck 2000) and the energy deposited per ionization is $20 \mathrm{eV}$ (Goldsmith 2001). The fractional abundance of $\mathrm{CO}$ is taken to be $10^{-4}$. The algorithm requires inputs of dust temperature (discussed in the previous paragraph), local velocity dispersion $(\delta v)$, column density, and local density. We use the local density calculated from the density profile (Equation (1)) to be self-consistent with our dust temperature calculation. The local velocity and the column density are needed in order to calculate the level of radiative trapping in the molecular cooling lines. The local velocity dispersion (characterized by the Doppler $b$ parameter) was assumed to be $1 \mathrm{~km} \mathrm{~s}^{-1}$ throughout the calculation. This is a reasonable assumption based on the values of the velocity field found in UME10 (see Table 5 below).

We estimate the column density at a point of interest $\mathbf{r}$ within our simulation with the following line integral, for every sink $i$ present in the simulation at that time:

$$
N_{\text {column }, i}=\int_{\Delta l_{i}}^{\Delta l_{i}+2000 \mathrm{AU}} n_{i}(r) d \hat{\mathbf{r}},
$$

where $\Delta l_{i}$ is the distance from sink $i$ to $\mathbf{r}, n_{i}(r)$ is the density profile around sink $i$ calculated using Equation (1), and $d \hat{\mathbf{r}}$ indicates that the direction of integration is radial. Hence, we are essentially calculating the column density of gas along a line of length $2000 \mathrm{AU}$ starting at the point of interest and pointing away from the sink. The sink particle that gives the highest column density at the point of interest is used to calculate the column density and also the local density at that point within 
the simulation. The choice of highest column density is made to ensure that we are not calculating the column density from the tail of the distribution around a more distant sink particle (see UED09). The limit of integration was set at $2000 \mathrm{AU}$ to agree with the value used in UED09.

As in UME10, we impose a minimum temperature $T_{\min }$ to the gas. Hence, if the calculation of $T_{\text {gas }}$ produces a value lower than $T_{\min }$, we use $T_{\text {gas }}=T_{\min }$ instead. We consider the effects of changing the value of $T_{\min }$.

\section{THE SIMULATIONS}

We performed two new simulations, G05 and G10, which include all the dust energetics discussed in Section 2.1, but also add a more accurate treatment of the gas energetics described in more detail in UED09. In the following results section, we will refer to these new simulations as using a "complete energetics" algorithm. For comparison, we also include two simulations, I05 and D05, that were presented in UME10. Our initial conditions are identical to those described in UME10. The parameters of the simulations are given in Table 1. In the third column, $T_{\text {dust }}$ and $T_{\text {gas }}$ refer to the dust and gas temperature calculated using the complete energetics algorithm, while $T_{\mathrm{K}}$ is the actual temperature that we use for the gas. The letters I, D, and G stand for "isothermal," "dust," and "gas," respectively.

In all simulations, the initial density of our cloud is $\bar{\rho}=$ $4.75 \times 10^{-20} \mathrm{~g} \mathrm{~cm}^{-3}$, or $\bar{n}=1.22 \times 10^{4} \mathrm{~cm}^{-3}$ assuming $\mu=2.33$. This density is similar to the median average density $\left(\bar{n}=1.6 \times 10^{4}\right)$ of a well-studied sample of massive dense clumps (Wu et al. 2010). In simulations I05, D05, and G05, the minimum temperature of the gas is set at $T_{\min }=5 \mathrm{~K}$, which corresponds to an initial Jeans mass $M_{J}^{\text {init }}=0.617 M_{\odot}$. Sink particles are created at a threshold density of $\rho_{c}=2.822 \times$ $10^{-16} \mathrm{~g} \mathrm{~cm}^{-3}$, or $n_{c}=7.252 \times 10^{7} \mathrm{~cm}^{-3}$, which represents a contraction by a factor of 5942. The corresponding Jeans mass at $\rho=\rho_{c}$ is $M_{J}=0.008 M_{\odot}$. As in UME10, we adjust the particle mass such that each Jeans mass is resolved with 200 particles. The simulation starts with $64^{3}$ particles, but we allow two levels of particle splitting $\left(N_{\text {gen }}=2\right.$, see UME10). The effective number of particles is therefore $256^{3}$, the total mass of the system is $M_{\mathrm{tot}}=\left(256^{3} / 200\right) M_{J}=671.4 M_{\odot}$, and the box size is $L_{\text {box }}=\left(M_{\text {tot }} / \bar{\rho}\right)^{1 / 3}=0.984 \mathrm{pc}$. Our SPH code uses a standard cubic spline smoothing kernel. The individual smoothing lengths are adjusted dynamically so that each gas particle has about 50 neighbors.

All simulations use identical initial conditions. Particles are laid down on a $64^{3}$ cubic grid, and displaced in order to reproduce a Gaussian random density fluctuation with a $P(k) \propto k^{-2}$ power spectrum (Klessen et al. 1998; MES06). Initial velocities are then adjusted in order to reproduce a pure growing mode (MES06). In simulation G05, we set the minimum temperature to $5 \mathrm{~K}$ to allow a direct comparison with simulations I05 and D05 taken from UME10. However, most observations suggest $T_{\mathrm{K}} \geqslant 10 \mathrm{~K}$, except in well-shielded areas of dense cores. Although massive, dense clumps may in fact be quite cold before they form stars, we explore the effects of $T_{\min }$ with a second simulation with full energetics, G10, in which we used $T_{\min }=10 \mathrm{~K}$. We did not change the initial density $\bar{\rho}$ and the threshold density $\rho_{c}$. Doubling the temperature while keeping $\rho_{c}$ fixed increases the minimum Jeans mass by a factor of $2^{3 / 2}$, up to $0.0226 M_{\odot}$. Following the approach used in UME10, we decided to keep the same resolution for all simulations: 200 particles per Jeans mass. As a result, the particle mass and total
Table 2

Final State for all Simulations

\begin{tabular}{lrrccc}
\hline \hline Run & $\begin{array}{c}t_{\text {final }} \\
\left(t_{\mathrm{ff}}\right)\end{array}$ & $N_{\text {sinks, final }}$ & $\begin{array}{c}M_{\text {sink, } \max } \\
\left(M_{\odot}\right)\end{array}$ & $f_{\text {sinks, final }}$ & SFR $_{\mathrm{ff}}$ \\
\hline I05 & 2.5 & 3429 & 0.50 & $60 \%$ & 0.24 \\
D05 & 2.5 & 74 & 20.8 & $50 \%$ & 0.20 \\
G05 & 2.4 & 118 & 20.8 & $46 \%$ & 0.19 \\
G10 & 2.4 & 365 & 24.0 & $47 \%$ & 0.20 \\
\hline
\end{tabular}

mass increase by a factor or $2^{3 / 2}$, and the box size increases by a factor of $2^{1 / 2}$. The total mass $M_{\mathrm{tot}}$, box size $L_{\mathrm{box}}$, the initial Jeans mass $M_{J}^{\text {init }}$, Jeans mass $M_{J}$ at sink formation, and accretion radius $r_{\text {acc }}$ are listed in Table 1 .

In all simulations, the first particle splitting occurs at a density $\rho=5.80 \bar{\rho}$, the second one at density $\rho=371 \bar{\rho}$, and sink formation starts at density $\rho=\rho_{c}=5942 \bar{\rho}$. The first sinks formed have an initial mass $M \approx M_{J}$. Sinks formed afterward will tend to be initially more massive since gas heating by the first sinks increases the Jeans mass. The initial free-fall time in our simulations is $t_{\mathrm{ff}}=9.64 \times 10^{12} \mathrm{~s}=3.06 \times 10^{5} \mathrm{yr}$. We run our simulations for a few free-fall times until the most massive sink particle in the simulation reaches $M \approx 21 M_{\odot}$. We halt the simulations at this point since the luminosity from massive stars will produce significant ionizing photons. These photons will then dominate the evolution of the simulation as seen in Dale et al. (2005).

\section{RESULTS}

\subsection{Fragmentation and Sink Formation}

Figure 1 shows the fractional mass distribution of the gas and sink particles as a function of time for the various simulations. The mass fraction in gas and in sinks is nearly identical for all runs with feedback (D05, G05, and G10). In all cases, the transition from gas- to sink-dominated mass fraction takes place around $t=2.45 t_{\mathrm{ff}}$, while for run $\mathrm{I} 05$ this transition takes place earlier, at $t=2.36 t_{\mathrm{ff}}$. By $t=2.4 t_{\mathrm{ff}}$, we have formed 74 sinks in run D05 and 118 in run G05. The slower formation of sink particles in run D05 for which $T_{\mathrm{K}}=T_{\text {dust }}$ is due to the fact that the dust temperature is usually larger than the gas temperature, and setting the gas temperature to that higher value tends to inhibit the formation of sinks. We discuss this in more detail in Section 4.4 .

It is interesting to compare runs G05 and G10. As we explained in Section 3, the volume simulated in run G10 is larger than the one simulated in run G05 by a factor of $2^{3 / 2}$. Hence, because run G05 formed 118 sinks by $t=2.4 t_{\mathrm{ff}}$, we would expect run G10 to form about 334 sinks just because of its increased mass, not accounting for the effect of temperature. Run G10 actually forms 365 sinks by $t=2.4 t_{\mathrm{ff}}$, within $8 \%$ of our prediction. The right axis in the bottom panel of Figure 1 has been rescaled by a factor of $2^{3 / 2}$ compared to the third panel to allow a direct visual comparison. Sinks form faster in run G10 than run G05, but eventually the number of sinks per unit volume becomes comparable. Changing the minimum temperature affects the formation of the first sinks. But once several sinks have formed, the minimum temperature becomes irrelevant, because feedback heating by sinks raises the gas temperature above $10 \mathrm{~K}$ in regions where the next sinks form, as we show in Section 4.4 below.

In Table 2, we compare the final state of all four simulations. The third, fourth, and fifth columns give the number of sinks, maximum sink mass, and mass fraction in sinks at the final time, 


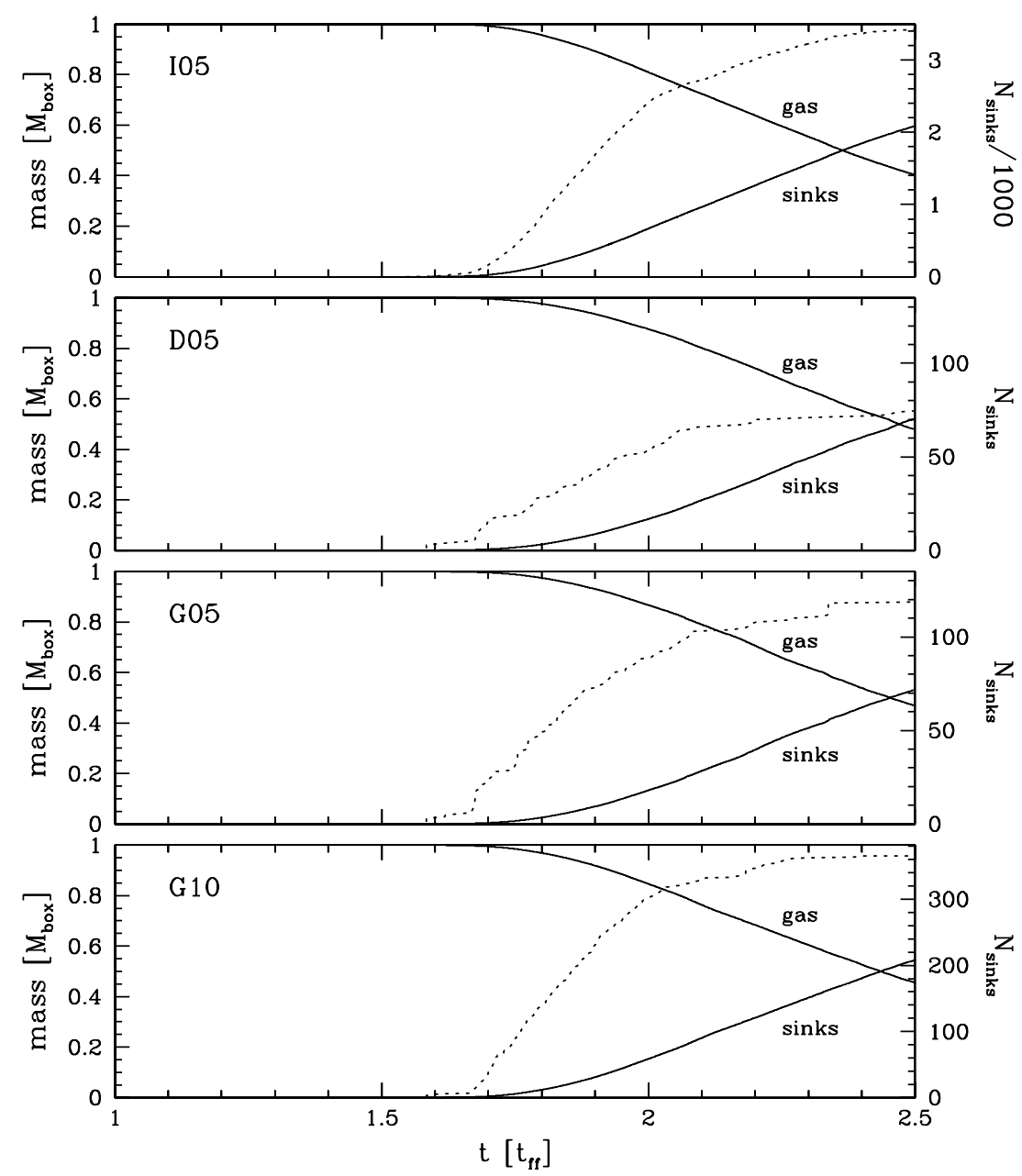

Figure 1. Evolution of the mass fraction in gas and sinks, and the number of sinks, for all four simulations. Top panel: run I05; second panel: run D05; third panel: run G05; and bottom panel: run G10. Top solid lines show the mass fraction in gas. Bottom solid lines show the mass fraction in sinks. Dotted lines with the scales on the right axes show the number of sinks. The scales of the right axes in the first and fourth panels are different from those in the second and third panel. The one in the fourth panel is larger than the one in the third panel by a factor of $2^{3 / 2}$ to account for the increased volume.

respectively. Comparing runs D05 and G05, which both have a total mass of $671 M_{\odot}$, we find that fewer sinks are formed in run D05, but the gas that is prevented from forming new sinks ends up accreting onto the existing sinks, so the total mass in sinks is roughly the same at the end of the simulations.

Figure 2 shows the distribution of sink and gas particles in our simulation box at $t=2.4 t_{\mathrm{ff}}$ for run G05. We chose this time because this is when a substantial fraction of the total number of final sinks have formed and there is also a significant amount of gas remaining, as seen in Figure 1. The morphology of the gas shown in this figure is similar to that seen in similar figures in UME10. There are fewer sinks formed compared to the isothermal simulation I05, but there are more compared to the simulation with only dust heating D05. The distribution of sink particles near the intersection of filaments is similar to what is seen in Bate (2009).

The sinks form in close proximity. To quantify this, starting with sink 2 , every time a sink formed, we calculated the distance to the nearest existing sink. The resulting distributions are shown as solid histograms in Figure 3. There is a "natural" separation $\Delta r=2 r_{\text {acc }}$, which comes from the method used for forming sinks. The algorithm converts gaseous spheres of radius $r_{\text {acc }}$ into sinks. When a dense region fragments into several sinks, the minimum separation is of order $2 r_{\text {acc }}$, corresponding to spheres of gas that are in contact. Also, when a new sink forms near an existing one, the separation tends to be of order $2 r_{\text {acc }}$ : the gas density profiles near sinks (Section 4.3) show that the density decreases with increasing radius; hence the gas located closest to the existing sink will be the first to reach the threshold density $\rho_{c}$. The large separations seen in Figure 3 correspond to sinks that were the first to form in a gas clump that was located away from all other clumps where sinks were already present. Although there is a physical reason for sinks to form close to one another, the particular value of $2 r_{\text {acc }}$ has no physical meaning. The parameter $r_{\text {acc }}$ is adjustable, and so is the density threshold $\rho_{c}$. Only the combination $r_{\text {acc }}^{3} \rho_{c}$ has a physical meaning, since it determines the Jeans mass $M_{J}$. We could have used a larger value of $r_{\text {acc }}$ and a correspondingly smaller value of $\rho_{c}$. Sinks would have formed farther apart, but gravity would have brought them together, and the end result would have been essentially the same. In very dense regions, separations between sinks can reach values as low as $10-20 \mathrm{AU}$. They are clearly much closer than they were when they formed.

Although some sinks move closer together, others move apart. The final distribution of separations is broad and skewed (dashed histograms in Figure 3), indeed bimodal. By the end of simulations G05 and G10, the mean separation is $4740 \mathrm{AU}$ and $4940 \mathrm{AU}$, respectively, with final median separations of 


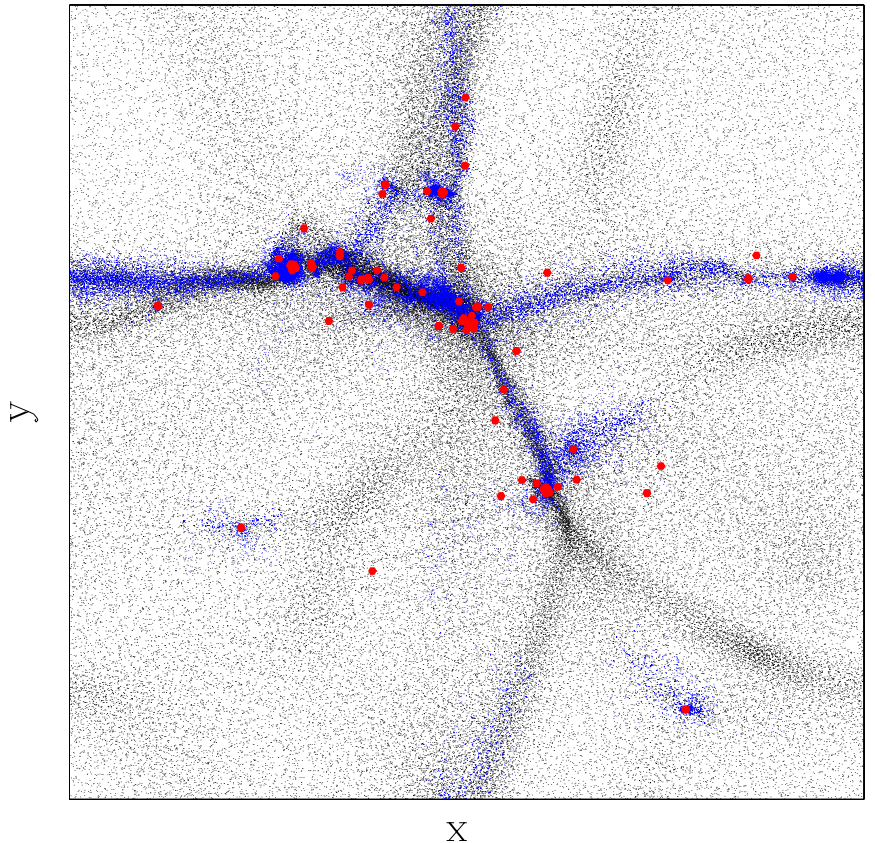

Figure 2. $X Y$ position plot of sinks and gas particles at $t=2.4 t_{\mathrm{ff}}$ for run $\mathrm{G} 05$. Black and blue dots indicate gas particles. Blue dots are gas particles which have undergone one particle splitting. Red dots are sinks. The box is $0.984 \mathrm{pc} \times$ $0.984 \mathrm{pc}$.

only $700 \mathrm{AU}$ and $556 \mathrm{AU}$, respectively. The medians are much smaller than even the projected median separations in nearby clusters of 14,800 AU (Gutermuth et al. 2009). The median separations in both simulations increased by a factor of three or more between $t / t_{\mathrm{ff}}=2.2$ and 2.4 , so further evolution may lead to larger mean separations. In addition, the overall distributions, especially for G10, do resemble the observations (e.g., Figure 2 of Gutermuth et al. 2009) in having a tail toward large separations. Given that the confusion limit of the observations is about $3000 \mathrm{AU}$, they would not resolve the very close pairs. Indeed, the peak of the distribution below $100 \mathrm{AU}$ might be interpreted as binaries and multiple systems.

\subsection{Evolution of Luminosity and Star Formation Efficiency}

Extragalactic observers employ a relation between total farinfrared luminosity and star formation rate to estimate star formation rates in dusty starbursts (Kennicutt 1998):

$$
\begin{aligned}
\operatorname{SFR}\left(M_{\odot} \mathrm{yr}^{-1}\right) & =4.5 \times 10^{-44} L_{\mathrm{FIR}}\left(\mathrm{erg} \mathrm{s}^{-1}\right) \\
& =1.7 \times 10^{-10} L_{\mathrm{FIR}}\left(L_{\odot}\right) .
\end{aligned}
$$

For convenience, we express this K98 relation as $\mathrm{SFR} / L=1.7 \times 10^{-4} M_{\odot} \mathrm{Myr}^{-1} L_{\odot}{ }^{-1}$ or $L / \mathrm{SFR}=5.9 \times$ $10^{3} L_{\odot}$ Myr $M_{\odot}{ }^{-1}$. A more recent calibration using the initial mass function (IMF) of Kroupa (2002) yields a slightly slower $\mathrm{SFR}$, or higher $L / \mathrm{SFR}=6.9 \times 10^{3} L_{\odot} \mathrm{Myr} M_{\odot}{ }^{-1}$.

$\mathrm{Wu}$ et al. (2005) have used this relation for massive dense clumps in our galaxy, finding a similar relation between $L_{\text {FIR }}$ and the line luminosity of dense gas tracers like HCN for individual clumps as was found in starburst galaxies by Gao \& Solomon (2004), as long as $L_{\text {FIR }}$ is above about $10^{4.5} L_{\odot}$. However, Krumholz \& Tan (2007) noted that the most massive stars, and hence the luminosity, take a considerable time to build up during cluster formation. Using an analytical prescription for star formation, they plotted $L /$ SFR versus time, finding values 1-2 orders of magnitude below the extragalactic relation for times less than 1 Myr.

In Figure 4, we show how the total luminosity $L_{*}^{\text {tot }}$, total sink mass $M_{*}^{\text {tot }}$, time-averaged star formation rate $\langle\mathrm{SFR}\rangle \equiv M_{*}^{\text {tot }} / t$, and $L_{*}^{\text {tot }} /\langle$ SFR $\rangle$ vary with time in our simulations. The build-up of stellar mass and luminosity is quite rapid, but the luminosity lags the mass. The calculations were stopped at $2.5 t_{\mathrm{ff}}$ or
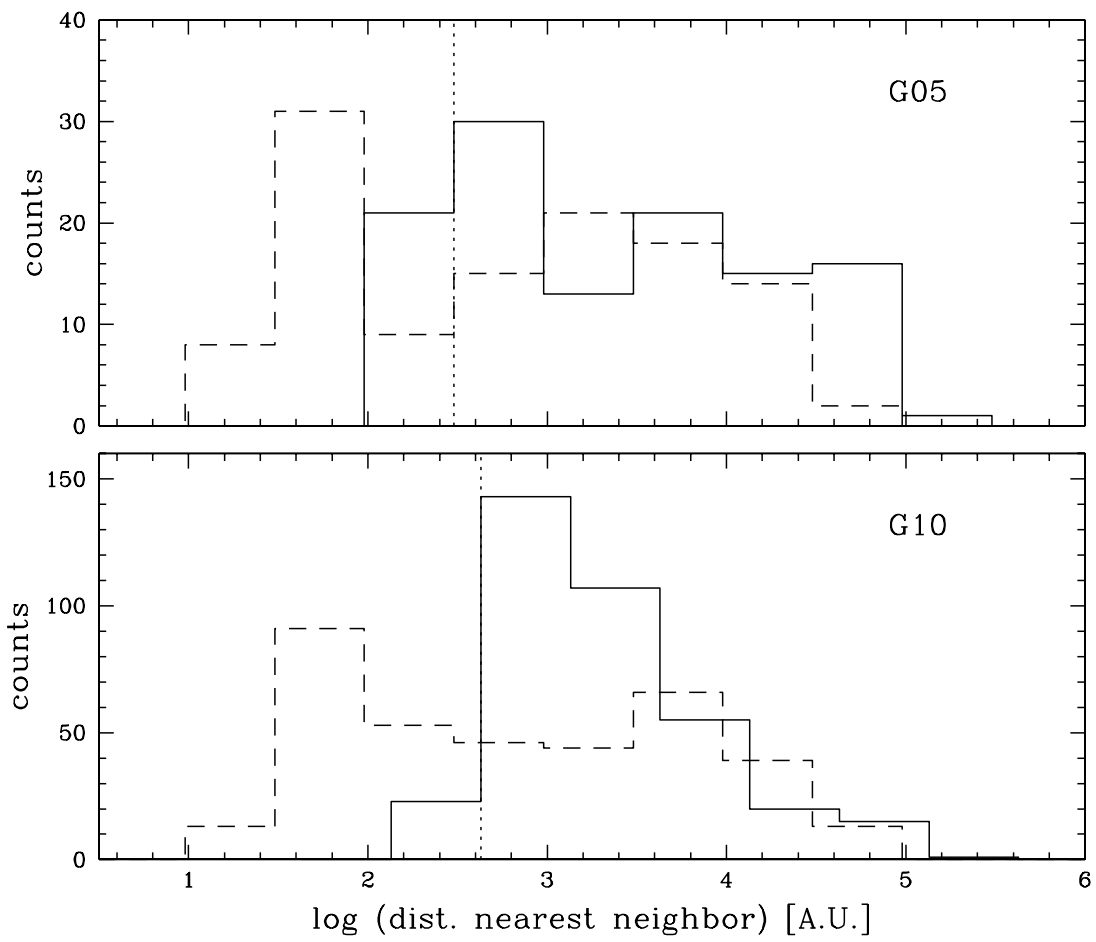

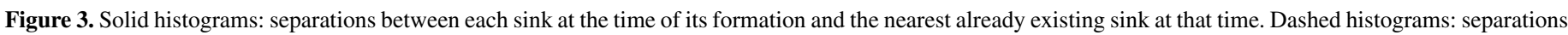
between each sink and its nearest neighbor at the end of the simulation. Top panel: run G05; bottom panel: run G10. Dotted lines indicate the value of $2 r_{\text {acc }}$. 

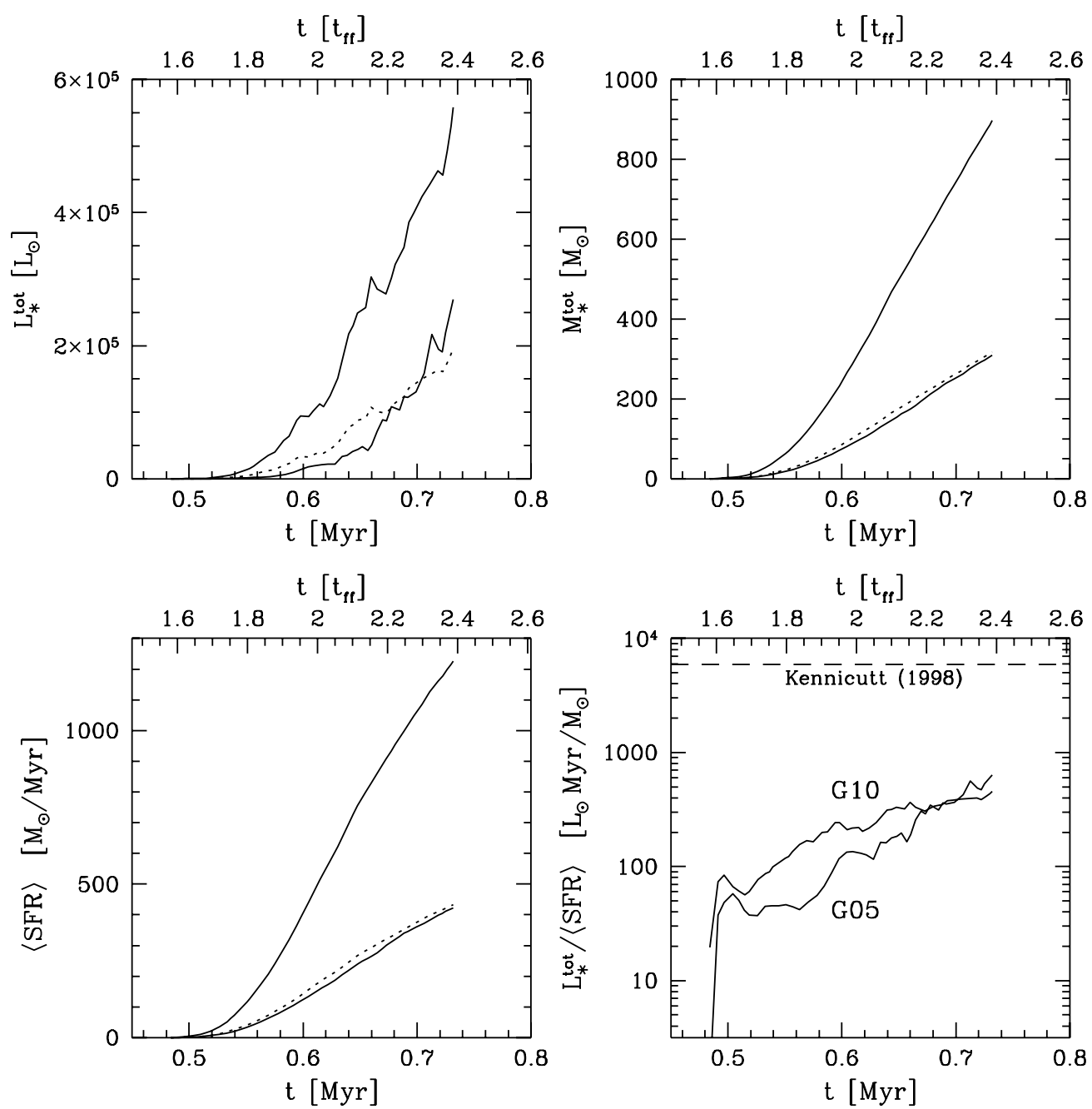

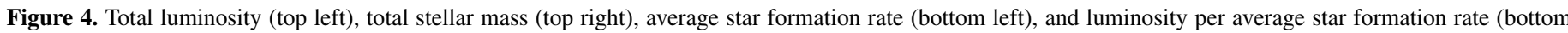

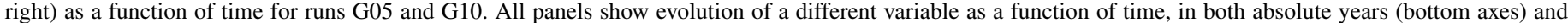

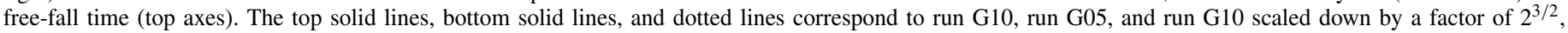
respectively. Also plotted in the bottom right panel as a horizontal line labeled (Kennicutt 1998) is the relation given in Equation (3).

$0.75 \mathrm{Myr}$, before the peak of star formation (see Figure 4). Both the sink formation rate and the mass accretion rate onto sinks are still increasing at the end of the simulation (see Figure 1). At that time, $L_{*}^{\text {tot }} /\langle\mathrm{SFR}\rangle$ lies a factor of 10 below the K98 relation. When the total luminosity first exceeds $10^{5} L_{\odot}$, the value of $L_{*}^{\text {tot }} /\langle\mathrm{SFR}\rangle$ lies about a factor of 20 below the K98 relation. The values of $L_{*}^{\text {tot }}, M_{*}^{\text {tot }}$, and $\langle\mathrm{SFR}\rangle$ are larger for run G10 simply because the volume simulated is larger. To allow a direct comparison with run G05, we plot the results for run G10, scaled down by a factor of $2^{3 / 2}$ (dotted line). The results are very similar to the ones for run G05.

These results confirm the prediction by Krumholz \& Tan (2007) that the K98 relation will underestimate the SFR for an individual cluster at early times, but the discrepancies are somewhat less than they found. If star formation has proceeded for a few free-fall times, or about $1 \mathrm{Myr}$, the K98 relation improves for an individual clump. However, there must still be sufficient dust to convert most of the luminosity into far-infrared radiation. Once ionizing radiation turns on, $L_{\mathrm{FIR}}$ may become a better tracer of the SFR. Vutisalchavakul \& Evans (2012) found agreement to a factor of two between SFRs calculated from $L_{\text {FIR }}$ and radio continuum emission for a sample of massive dense clumps.
The last column of Table 2 shows the star formation efficiency per free-fall time, $\mathrm{SFR}_{\mathrm{ff}}$, defined in Krumholz \& McKee (2005). It is essentially the ratio $f_{\text {sinks, final }} / t_{\mathrm{ff}}$. The values are similar for all runs, even though the number of sink particles is higher in the new simulations compared to the simulation with only dust heating energetics. The values in Table 2 assume that all mass entering the sink winds up in the star. As noted earlier, this almost certainly overestimates the stellar mass by factors of 2-3, suggesting values of $\mathrm{SFR}_{\mathrm{ff}}$ of $0.07-0.10$. Star formation efficiencies in massive dense clumps are not well constrained, but are certainly much lower. Using the numbers for the mean $L_{\mathrm{FIR}} / M_{\mathrm{vir}}$ for massive dense clumps from $\mathrm{Wu}$ et al. (2010) and Equation (3), along with the free-fall time at the mean density of these clumps $(0.27 \mathrm{Myr})$, yields values of $\mathrm{SFR}_{\mathrm{ff}}$ around 0.006. If $L_{\mathrm{FIR}}$ underestimates the SFR in those clumps by a factor of 20 , the values would agree better with those in Table 2, but more likely the $\mathrm{SFR}_{\mathrm{ff}}$ is lower than found in our simulation. Overly fast star formation is a common feature of simulations that do not include means to slow down the star formation process. Even recent simulations with radiative feedback, turbulence, and outflows (Krumholz et al. 2012) produce very similar values of $\mathrm{SFR}_{\mathrm{ff}}$ to those in Table 2. 


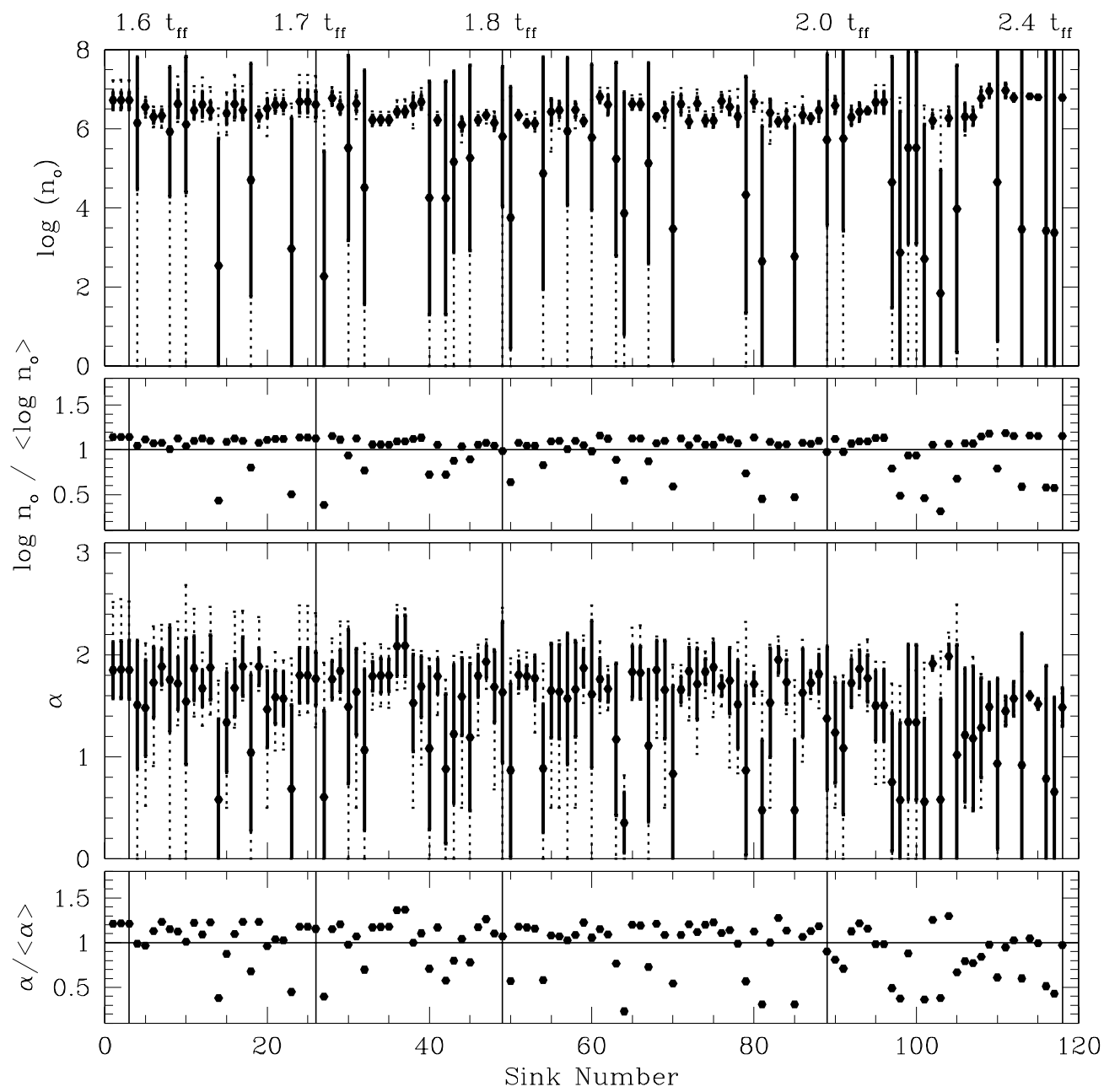

Figure 5. Density profile parameters for all 118 sinks formed in simulation G05. Values of density profile parameters, $\alpha$ and $n_{o}$, are shown for the sinks. Error bars shown with a solid line indicate the standard deviation for each individual sink. Error bars shown with a dotted line indicate the minimum and maximum value that $\alpha$ and $n_{o}$ take during the course of the simulation.

Table 3

Density Profile around Sinks

\begin{tabular}{ccc}
\hline \hline Run & $\langle\alpha\rangle$ & $\left\langle\log \left(n_{o} / \mathrm{cm}^{-3}\right)\right\rangle$ \\
\hline D05 & $1.7 \pm 0.4$ & $6.5 \pm 0.3$ \\
G05 & $1.7 \pm 0.4$ & $6.4 \pm 0.3$ \\
\hline
\end{tabular}

\subsection{Density Profiles around Sinks}

We calculate the density profile around each sink at each time step. The density profile will change around a sink as it moves within the cloud, gathering gas particles and/ or as gas particles accrete irregularly onto it. In Section 2 we defined the density parameters, $n_{o}$ and $\alpha$, of the density profile, Equation (1). Figure 5 shows the average values of the density profile parameters, $\alpha$ and $n_{o}$, for all the sinks that formed in run G05. We only included sinks that have accretion rates $\dot{M}>10^{-8} M_{\odot} \mathrm{yr}^{-1}$, because sinks with lower accretion rates have too few neighboring gas particles to allow for an accurate determination of the profile. We summarize the results in Table 3, together with the results for run D05, taken from UME10. The values are similar for both runs. This suggests that the density profile surrounding the individual sinks is not strongly affected by the energetics algorithm used.
Groups studying young star-forming cores have calculated density profiles from observations. Comparing our average density profile values to those observationally derived values, we find excellent agreement with our values of $\alpha$ and $n_{0}$. Shirley et al. (2002) studied Class 0 cores and found $\langle\alpha\rangle=1.63 \pm 0.33$ and a typical value of $\alpha=1.8 \pm 0.1$ if they ignored two sources with aspherical emission contours. Young et al. (2003) studied Class I cores and found $\langle\alpha\rangle=1.6 \pm 0.4$. Our simulations are consistent with either of these values for $\alpha$. The values of $\log n_{o}$ derived from these two studies are $\left\langle\log \left(n_{o} / \mathrm{cm}^{-3}\right)\right\rangle=6.1 \pm 0.2$ (Shirley et al. 2002) and $\left\langle\log \left(n_{o} / \mathrm{cm}^{-3}\right)\right\rangle=5.4 \pm 0.5$ (Young et al. 2003). Our value of $\left\langle\log \left(n_{o} / \mathrm{cm}^{-3}\right)\right\rangle$ is $6.4 \pm 0.3$, including only points with accretion rates greater than $10^{-8} M_{\odot} \mathrm{yr}^{-1}$. These values tend to agree better with the results for the Class 0 core study of Shirley et al. (2002), suggesting that most of the sinks are reflecting early stages in star formation.

\subsection{Evolution of Temperature, Density, and Velocity}

Figure 6 shows the density and temperature evolution of gas particles in our simulations. The behavior of the gas particles in these figures resembles what was seen in similar figures in UME10. For example, at early times the temperatures of the gas particles are confined to low values at all densities. However, as the simulation evolves and more sink particles form, the gas particles are heated and tendrils of gas particles appear 

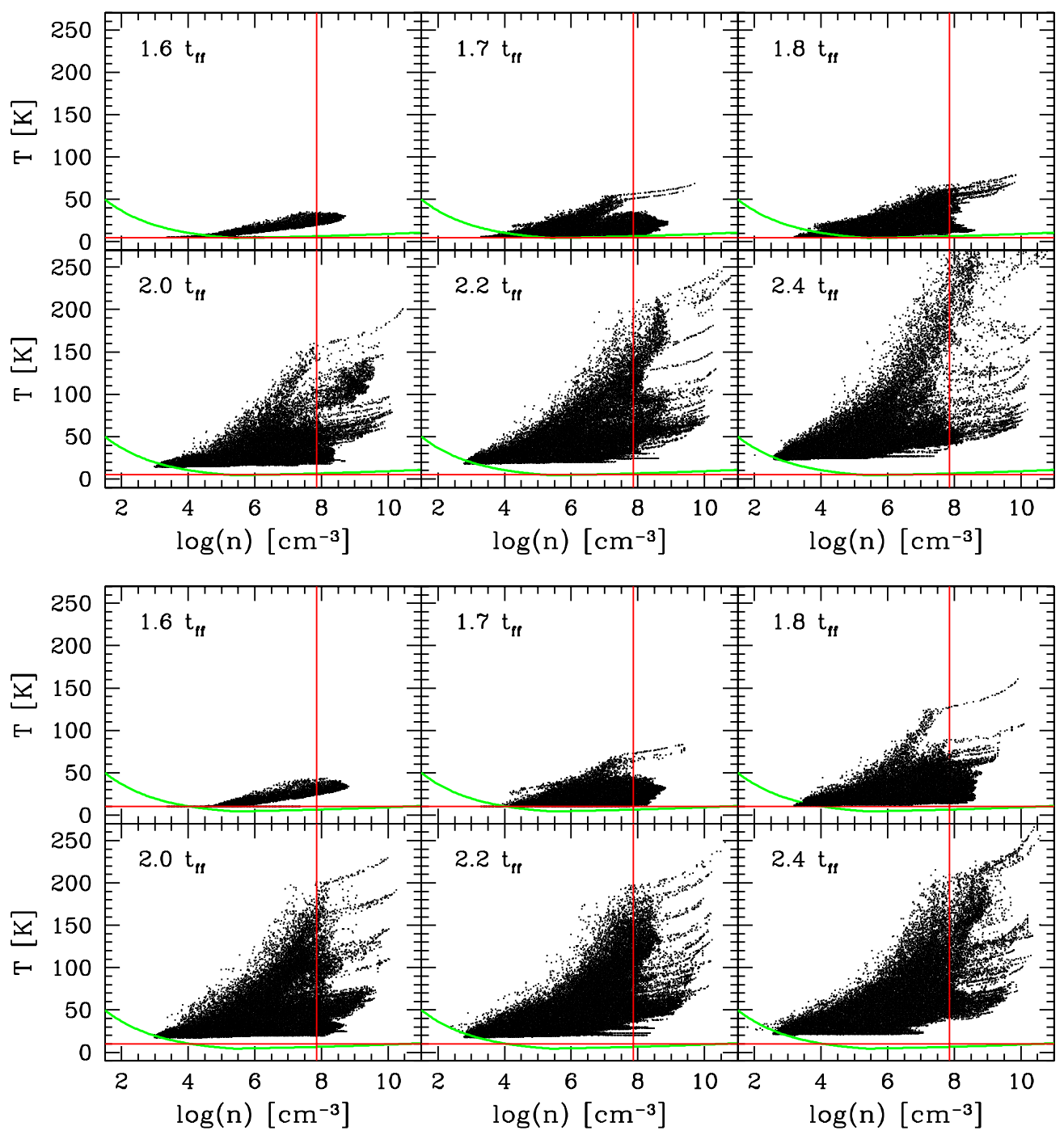

Figure 6. Temperature and density of gas particles as a function of time for runs G05 (top six panels) and G10 (bottom six panels), at various times, as indicated. The green line shows the equation of state given by Larson (2005). The horizontal and vertical red lines show the minimum temperature $T_{\min }$ and threshold density $n_{c}$ for sink formation, respectively. The statistics of the temperature are listed in Table 4.

in Figure 6. They are reaching toward high-density and hightemperature regimes. These tendrils show the behavior of the gas density and temperature near luminous sink particles. Another interesting feature seen in this figure is the slight increase of the floor of the temperature distribution as a function of time. This feature of an increasing temperature floor was also seen in run D05 (UME10). In Figure 6, we also plot the equation of state given by Larson (2005). As in run D05 (UME10), the gas does not follow this equation. This is not surprising because the equation of state of Larson (2005) does not take into account stellar heating. This has been confirmed to be an important effect in Krumholz et al. (2007), Offner et al. (2009), and UME10.

The horizontal and vertical red lines in Figure 6 show the minimum temperature $T_{\min }$ and the density threshold $\rho_{c}$, respectively. Gas particles located on the right of the vertical line, in regions where sinks form, all have temperatures significantly larger than $T_{\min }$. These particles do not turn into sinks because their high temperatures make them unbound (see Equation (13) in MES06). This explains the results that were presented in Figure 1 and Table 2. Increasing the minimum temperature from $5 \mathrm{~K}$ to $10 \mathrm{~K}$ might affect the formation of the first few sinks, but once these sinks have formed and started reheating the gas, the formation of subsequent sinks is unaffected by the particular value of $T_{\min }$, because the gas temperature in regions of sink formation is already much larger. This explains why the gas fraction drops at essentially the same rate in simulations G05 and G10 (Figure 1), and why both simulations form roughly the same number of sinks per unit volume. Judging from Figure 6, it would take a minimum temperature of order $50 \mathrm{~K}$ or more to make a difference.

Most of the cores in our simulations are located inside dense filaments. The few cores located in low-density regions actually formed inside filaments, and were later ejected by three-body encounters (MES06). This concentration of cores can explain the large gas temperatures found in these regions (even though more cores mean more competition for accreting gas from the same region, possibly leading to lower accretion luminosities). The spatial distribution of cores is a consequence of the assumed periodic boundary conditions. Heitsch et al. (2008) performed simulations of cloud fragmentation with isolated boundary conditions, and showed that these initial conditions lead to stronger initial fragmentation in the early stages, and a more distributed core formation at late stages. Observations are, however, finding strong concentrations of cores and protostars 
Table 4

Particle Statistics for Runs with Heating

\begin{tabular}{|c|c|c|c|c|c|c|c|}
\hline Run & $\begin{array}{l}\text { time } \\
\left(t_{\mathrm{ff}}\right)\end{array}$ & $\begin{array}{l}T_{\text {gas }} \\
(\mathrm{K})\end{array}$ & $\begin{array}{c}T_{\text {dust }} \\
(\mathrm{K})\end{array}$ & $\begin{array}{c}\log n \\
\left(\mathrm{~cm}^{-3}\right)\end{array}$ & $N_{\text {sinks }}$ & $f_{\text {sinks }}$ & $\begin{array}{c}\max \\
\left(M_{\text {sink }}\right)\end{array}$ \\
\hline D05 & 1.6 & $5.40 \pm 1.91$ & $5.40 \pm 1.91$ & $4.65 \pm 0.67$ & 3 & $0.02 \%$ & 0.07 \\
\hline D05 & 1.7 & $9.28 \pm 3.84$ & $9.28 \pm 3.84$ & $5.02 \pm 1.08$ & 16 & $0.4 \%$ & 0.72 \\
\hline D05 & 1.8 & $14.8 \pm 6.08$ & $14.8 \pm 6.08$ & $5.12 \pm 1.12$ & 28 & $2.4 \%$ & 2.11 \\
\hline D05 & 2.0 & $24.5 \pm 9.66$ & $24.5 \pm 9.66$ & $5.05 \pm 1.15$ & 53 & $12 \%$ & 7.99 \\
\hline D05 & 2.2 & $33.0 \pm 14.3$ & $33.0 \pm 14.3$ & $4.88 \pm 1.16$ & 70 & $28 \%$ & 12.40 \\
\hline D05 & 2.4 & $48.3 \pm 29.7$ & $48.3 \pm 29.7$ & $4.76 \pm 1.21$ & 71 & $45 \%$ & 17.99 \\
\hline G05 & 1.6 & $5.64 \pm 1.84$ & $4.50 \pm 2.20$ & $4.63 \pm 0.68$ & 3 & $0.03 \%$ & 0.06 \\
\hline G05 & 1.7 & $7.61 \pm 4.14$ & $9.64 \pm 3.89$ & $5.16 \pm 1.15$ & 26 & $0.5 \%$ & 0.78 \\
\hline G05 & 1.8 & $10.8 \pm 7.22$ & $14.9 \pm 5.94$ & $5.41 \pm 1.20$ & 49 & $2.8 \%$ & 1.7 \\
\hline G05 & 2.0 & $20.6 \pm 10.8$ & $25.9 \pm 10.6$ & $5.40 \pm 1.19$ & 89 & $14 \%$ & 7.9 \\
\hline G05 & 2.2 & $28.2 \pm 18.1$ & $33.6 \pm 17.8$ & $5.19 \pm 1.21$ & 108 & $30 \%$ & 12.8 \\
\hline G05 & 2.4 & $35.6 \pm 25.6$ & $43.0 \pm 25.4$ & $4.89 \pm 1.18$ & 118 & $46 \%$ & 20.8 \\
\hline G10 & 1.6 & $10.2 \pm 1.67$ & $5.35 \pm 2.60$ & $4.65 \pm 0.67$ & 5 & $0.03 \%$ & 0.12 \\
\hline G10 & 1.7 & $11.4 \pm 4.24$ & $11.8 \pm 4.78$ & $5.21 \pm 1.11$ & 37 & $0.6 \%$ & 2.22 \\
\hline G10 & 1.8 & $17.5 \pm 7.07$ & $19.6 \pm 7.11$ & $5.63 \pm 1.26$ & 138 & $3.1 \%$ & 6.08 \\
\hline G10 & 2.0 & $26.7 \pm 12.8$ & $30.0 \pm 13.1$ & $5.76 \pm 1.22$ & 301 & $15 \%$ & 15.1 \\
\hline G10 & 2.2 & $33.1 \pm 18.2$ & $35.7 \pm 18.0$ & $5.57 \pm 1.22$ & 346 & $32 \%$ & 21.8 \\
\hline G10 & 2.4 & $39.6 \pm 22.6$ & $42.7 \pm 22.5$ & $5.41 \pm 1.24$ & 365 & $47 \%$ & 24.0 \\
\hline
\end{tabular}

Table 5

Statistics of Accretion onto Sinks

\begin{tabular}{lrcc}
\hline \hline Run & \multicolumn{1}{c}{$\mathcal{v}$} & $\begin{array}{c}v \\
\left(\mathrm{~km} \mathrm{~s}^{-1}\right)\end{array}$ & $\begin{array}{c}\dot{M} \\
\left(10^{-5} M_{\odot} \mathrm{yr}^{-1}\right)\end{array}$ \\
\hline D05 & $10.8 \pm 7.4$ & $9.2 \pm 8.2$ & $2.20 \pm 3.08$ \\
G05 & $8.2 \pm 5.9$ & $5.5 \pm 5.3$ & $1.65 \pm 2.32$ \\
G10 & $10.8 \pm 7.6$ & $6.5 \pm 6.0$ & $1.37 \pm 2.36$ \\
\hline
\end{tabular}

along narrow filaments (e.g., Hennemann et al. 2012), very similar to patterns seen in the simulations.

Table 4 shows various statistics for runs with heating (D05, G05, and G10), i.e., the average gas and dust temperatures, and average density (calculated by averaging over all gas particles), number of sink particles, percentage of mass in sink particles, and mass of the most massive sink particle, at different times. We find that the average gas and dust temperatures both increase throughout the simulations. This increase can be explained by the increasing number of sink particles and the increasing mass of the individual sink particles, both of which lead to more substantial radiation fields.

Since it is essentially the mass of sinks and accretion rate of gas onto sinks that determine the dust temperature, we expect the temperature of the gas being accreted to have little effect if it accretes supersonically, which is indeed the case (see Table 5 below). At all times $t \geqslant 1.7 t_{\mathrm{ff}}$ in both G05 and G10, the mean gas temperature is slightly cooler than the dust temperature. This is expected since some gas is at quite low densities $\left(n \sim 10^{3}\right.$ or $10^{4} \mathrm{~cm}^{-3}$ ) and the rest is no hotter than the dust temperature. The gas is fully equilibrated to the dust temperature only at quite high densities, such as $n>10^{6} \mathrm{~cm}^{-3}$. The dust temperature is initially fixed at $5 \mathrm{~K}$ for run G05 and $10 \mathrm{~K}$ for run G10, and it can change only when heating via nearby stellar radiation fields begins. Figure 7 illustrates these effects in more detail. It shows the histograms of the dust and gas temperature at various times for low-density $\left(n<10^{5} \mathrm{~cm}^{-3}\right)$ and high-density $\left(n>10^{5} \mathrm{~cm}^{-3}\right.$ ) gas. Except for the first time step shown, the dust temperature is higher than the gas temperature at low densities (red histograms). It also shows that the dust and gas temperatures are nearly equal at high densities (black histograms) due to dust-gas collisional coupling. At $t=1.6 t_{\mathrm{ff}}$, the dust is cooler on average than the gas, for low-density gas (see also the first line of Table 4). At these early times, the dust has not yet been heated to high temperatures by forming stars. Because the dust temperatures are lower, the dominant heating source for lowdensity gas in our simulations is cosmic rays, which can raise the gas temperature above the dust temperature.

Figure 8 shows the dust-gas temperature difference as a function of density at various times for run G05. The contours indicate the region in parameter space, $T_{\text {dust }}-T_{\text {gas }}$ versus $\log n$, where the majority of the material is located. The gas is confined to the same region for most of the simulation, except during early times when the dust is heating up due to formation of luminosity sources. As seen before in Table 4 and Figure 7 during the earliest times, the gas temperature is hotter than the dust temperature because of the low intensity of the radiation field. At high densities $(\log n>8)$, the SPH gas particles tend to approach the horizontal line which indicates equal dust and gas temperature. At these high densities when the dust and gas temperatures are comparable, dust heating dominates all other forms of heating/cooling for the gas. This result was seen in UED09. By $2 t_{\mathrm{ff}}$, almost all the gas has $T_{\mathrm{K}}$ within $30 \%$ of $T_{\text {dust }}$, and a large fraction is even closer to equilibration. Figure 8 also shows that high dust temperatures are most likely to exist in high-density regions (as seen by the points), presumably due to the proximity of a nearby forming star.

Figures 7 and 8 show that neither the gas nor the dust is well described by isothermal or barotropic equations of state once protostars begin to heat their surroundings. There is a strong effect on the thermal behavior of the clump once protostars develop significant luminosity. That effect occurs during the sink formation process.

For each gas particle accreted onto a sink, we calculated the velocity $v$ of the particle relative to the sink, and the Mach number $\mathcal{M}=v / c_{s}$, where $c_{s}$ is the sound speed at the location of the sink. Table 5 shows the mean values of $v$ and $\mathcal{M}$, and also the mean values of the accretion rate $\dot{M}$, for all simulations with heating. Again, similar behavior of the sinks is seen for both G05 and G10, and also D05. On average, particles are being accreted supersonically. The sound speeds near sinks are comparable 

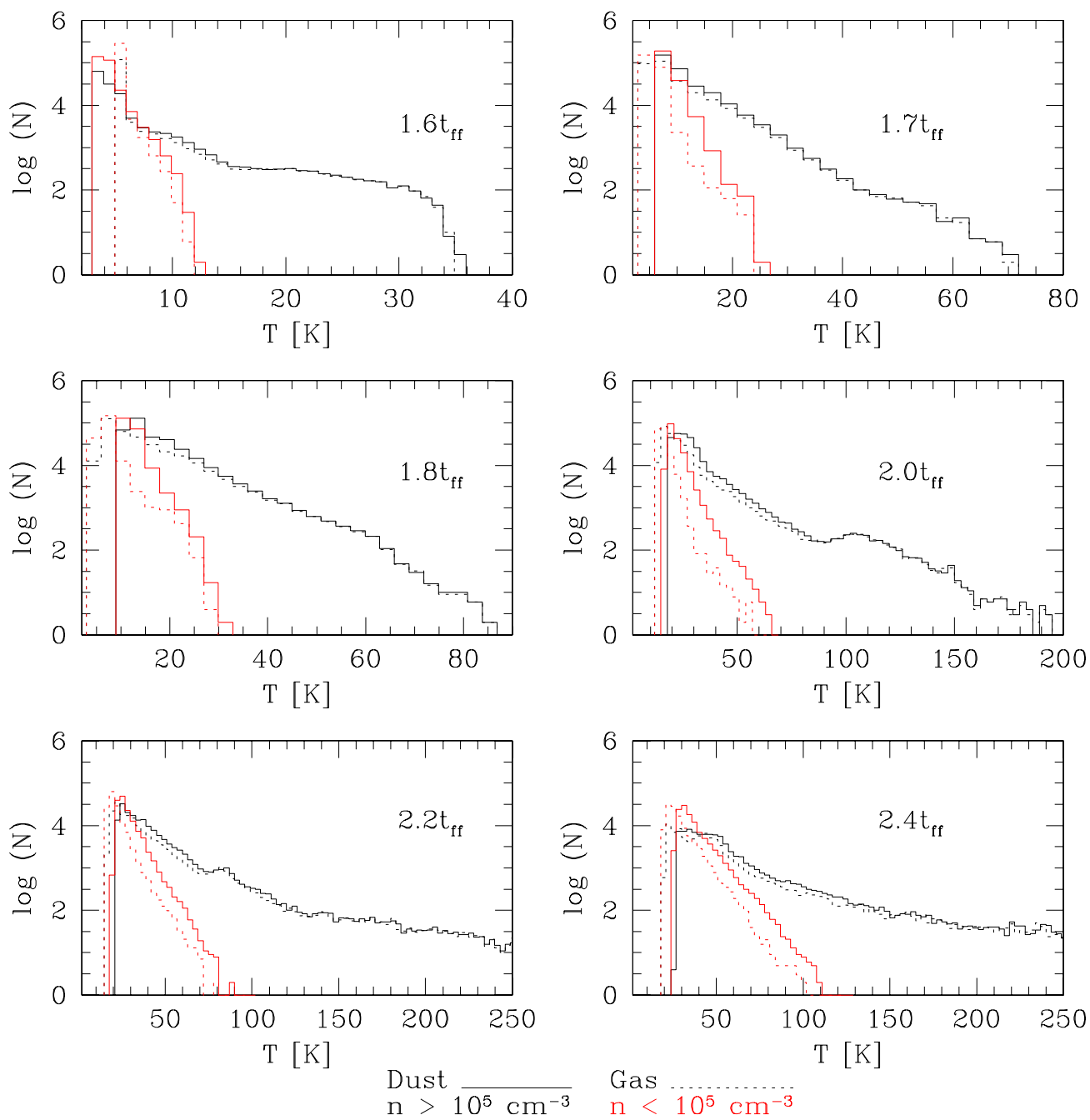

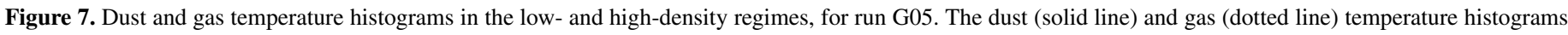
are plotted for low (red) and high (black) densities.

for runs G05 and G10, since, as we saw, the temperature in these regions greatly exceeds $T_{\min }$. Hence, the slightly larger accretion velocities for run G10 result in slightly larger Mach numbers. Compared to the runs with full energetics, run D05 has on average larger accretion rates, larger accretion velocities, and comparable Mach numbers. This run forms fewer sinks, but the total mass in sinks is the same (see Figure 1 and Table 2). Hence, the same mass is being accreted by fewer sinks, resulting in higher accretion rates. Also, fewer sinks mean that they are on average more massive. Since the accretion velocities are highly supersonic, gas particles accrete essentially at the escape velocity, which is larger for more massive sinks. However, the gas temperature, and consequently the sound speed, is also larger for run D05. As a result, the Mach numbers are comparable to the ones for runs G05 and G10.

These infall velocities are considerably higher than the assumed turbulent broadening (Doppler $b$ parameter is set to $1 \mathrm{~km} \mathrm{~s}^{-1}$ as discussed in Section 2.1). To the extent that these larger velocities allow for greater escape probabilities for photons in the primary cooling lines, the gas could be somewhat cooler than we calculate in the infalling gas around a sink. On the other hand, these are just the regions where the density becomes large enough to overwhelm gas cooling and couple the gas temperature to the dust temperature, so the effects are probably minimal.

\subsection{Mass Functions}

The Galactic Field Star IMF has been studied by many groups (see Salpeter 1955; Miller \& Scalo 1979; Scalo 1986; Kroupa 2002; Chabrier 2003). The cluster IMF has also been studied in young clustered, star-forming regions such as Orion (Hillenbrand 1997) and in more isolated star-forming regions in the Taurus (Briceño et al. 2002), Lupus (Comerón et al. 2009), and Chamaeleon (Alcala et al. 1997) molecular clouds. In a recent review paper, Bastian et al. (2010) found no significant variations in the IMF for present-day star formation.

In large-scale simulations of cluster formation, various treatments of thermal energetics were used. The earliest attempts assumed an isothermal equation of state (Klessen et al. 1998; MES06). In the work of MES06, we found that a properly resolved isothermal calculation could only produce very low mass fragments. The mass function in these simulations was log-normal with a peak that was determined by the resolution limit of the simulation. Other simulations attempted to address this issue by modifying the equation of state to account for the increase in optical depth in higher density gas. They used a barotropic equation of state in which the temperature of the gas increases at higher densities (Bate et al. 2003; Li et al. 2003; Bate 2005; Jappsen et al. 2005; Larson 2005; Bonnell et al. 2006; Clark et al. 2008). In more recent work, groups have 

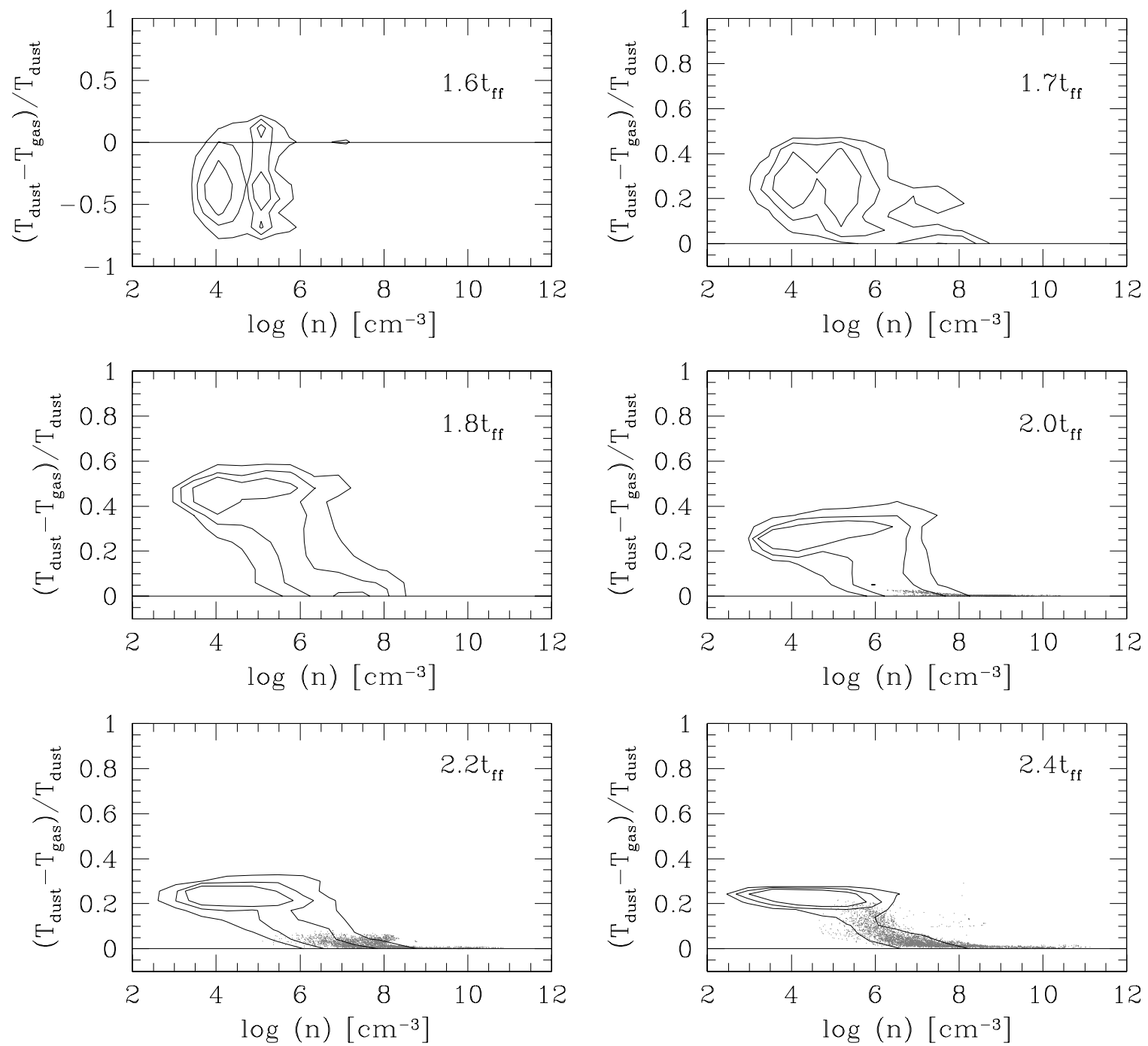

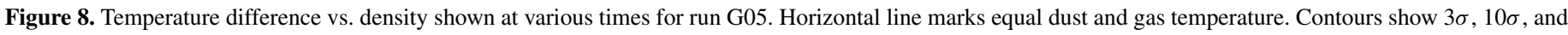
$20 \sigma$ contour levels of density of SPH gas particles. Dots indicate gas particles which have dust temperatures greater than $100 \mathrm{~K}$.

included various treatments of radiative transfer to more accurately account for the heating effect of the young stars on the gas (Krumholz et al. 2010; Bate 2009; Offner et al. 2009; Smith et al. 2009; UME10). Inclusion of the heating effect shifts the mass function to higher masses.

Figure 9 shows the mass functions of the sink particles at various times in the simulations G05 and G10. At early times, the mass function is dominated by accreting low-mass objects. As the simulations evolve, these low-mass sinks continue to accrete and become more massive. At later times, the formation of low-mass sinks becomes less frequent because the gas is now hotter and the Jeans mass is higher, causing a delay in sink formation until they have reached the new higher Jeans mass. Although there is a tendency for the mass function to shift its peak to higher masses with time, the IMF in simulation G10 becomes relatively stable after about $1.8 t_{\mathrm{ff}}$. The shift to higher masses relative to the isothermal calculation agrees with the results of the references given above.

We also plot the IMFs from Salpeter (1955), Chabrier (2003), and Kroupa (2002) in the last panel for each run. We normalize the mass functions using the maximum mass in the simulation (solid curves) and the total number of objects (dashed curves), both given in Table 2. The minimum mass used to create the IMFs was $0.08 M_{\odot}$ and $0.01 M_{\odot}$ for the Salpeter (1955) and Chabrier (2003)/Kroupa (2002) IMFs, respectively. Normalizing the mass functions based on the total number of objects highlights the fact that we form too few objects given the mass of the most massive sink in each simulation. The IMFs normalized to the maximum mass demonstrate that we can roughly reproduce the slope of the high-mass tail above masses of $\sim 1 M_{\odot}$.

As in UME10, our mass functions slightly overpredict the number of high-mass objects and miss a substantial fraction of low-mass objects. In UME10, we predicted that by including molecular cooling and cosmic-ray heating we would see a decrease in high-mass objects and an increase in intermediateto low-mass objects. This has turned out to be the case and can be seen in the top panel of Figure 10 where we show the mass functions for our three different simulations with a minimum temperature of $5 \mathrm{~K}$. We form more intermediate-mass objects, while still retaining the slope at higher masses.

In Table 6, we give the average and median sink masses in our simulations. We also give these values predicted by various empirical IMFs. We use a lower mass limit of $0.08 M_{\odot}$ for the Salpeter IMF and $0.01 M_{\odot}$ for the Kroupa and Chabrier IMFs. Including the complete energetics algorithm (G05 and 

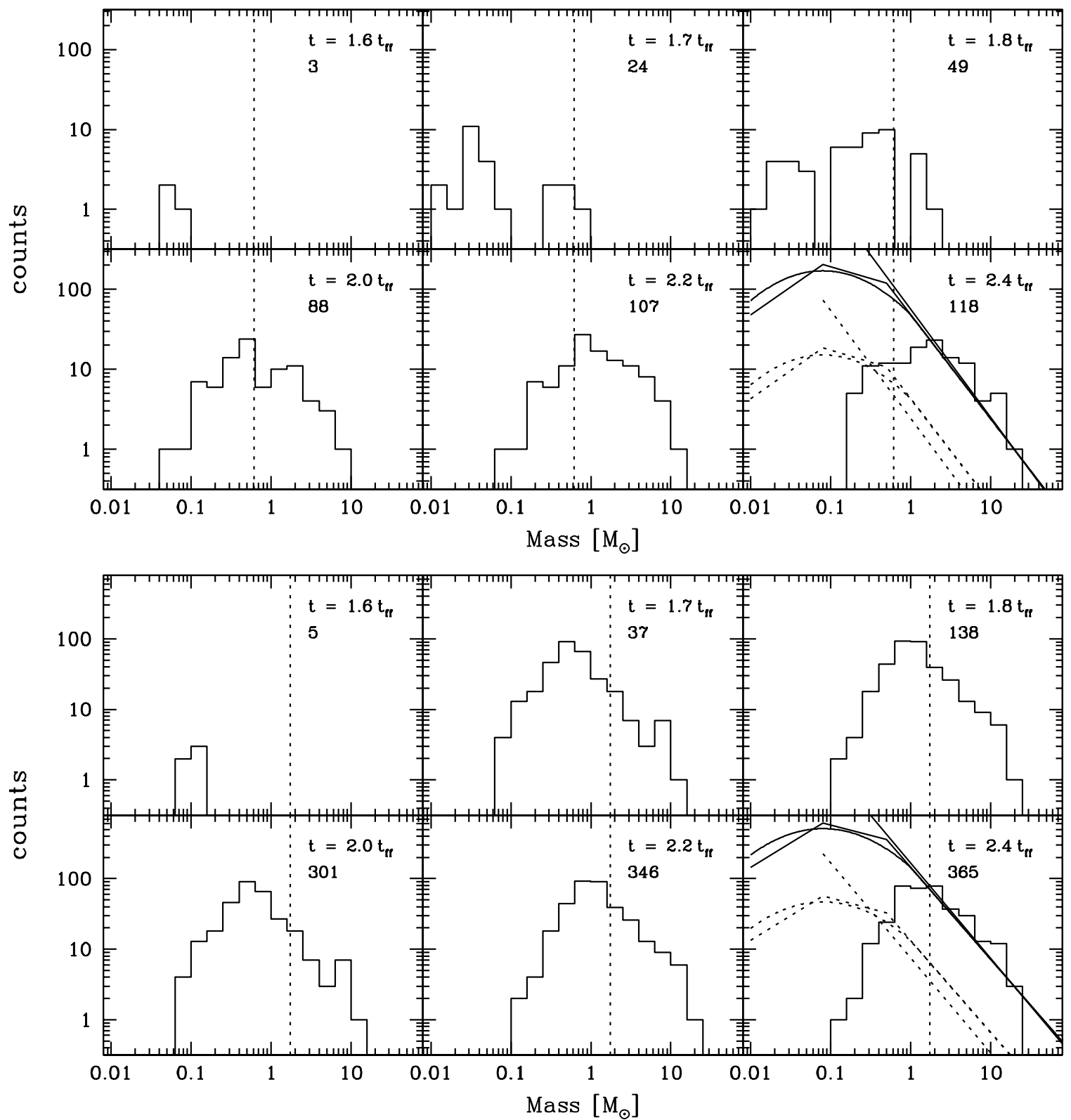

Figure 9. Mass histograms for runs G05 (top panels) and G10 (bottom panels), shown at different times. The time and number of sinks are indicated in each panel. The analytic mass functions of Salpeter (1955; straight line), Chabrier (2003; curved line), and Kroupa (2002; segmented line) are shown as solid lines when normalized to maximum mass object, and dashed lines when normalized to the total number of objects. Vertical dashed lines show initial Jeans mass in the simulation.

Table 6

Average and Median Masses

\begin{tabular}{lcc}
\hline \hline Run/Observations & Median & Average \\
\hline D05 & 2.26 & 4.63 \\
G05 & 1.63 & 2.65 \\
G10 & 1.50 & 2.46 \\
G10 $(\times 0.30)$ & 0.45 & 0.74 \\
\hline Salpeter $(1955)^{\mathrm{a}}$ & 0.13 & 0.26 \\
Chabrier $(2003)^{\mathrm{a}}$ & 0.10 & 0.31 \\
Kroupa $(2002)^{\mathrm{a}}$ & 0.15 & 0.34 \\
\hline
\end{tabular}

Notes. ${ }^{\text {a }}$ Median calculated using normalization to the total number of objects formed; average calculated using normalization to maximum mass object.

G10) decreases the resulting mean mass, compared to D05, as predicted. The change in the median mass shows a similar behavior. Although our median and average masses decrease, they still give values which are in disagreement with the empirical IMFs. Next, we perform a thought experiment to address this issue.

The regions around our sinks have density profiles similar to those of gas around Class 0 objects discussed in Section 4.3. Thus, it is reasonable to associate them with dense cores. As this material falls into the sinks, we have so far assumed that all the mass winds up in a star. Alves et al. (2007) claim that the dense core mass function is related to the stellar IMF, i.e., sharing the same shape, but shifted to higher masses. This is interpreted as a core-to-star efficiency factor of 30\%. Enoch et al. (2008) find a lower limit of $25 \%$ for the core-to-star formation efficiency. The missing mass is likely to be removed in molecular outflows driven by stellar winds (e.g., Dunham et al. 2010; Hansen et al. 2012). These outflows are not included in our simulation. To include this inefficiency, we scale the mass 


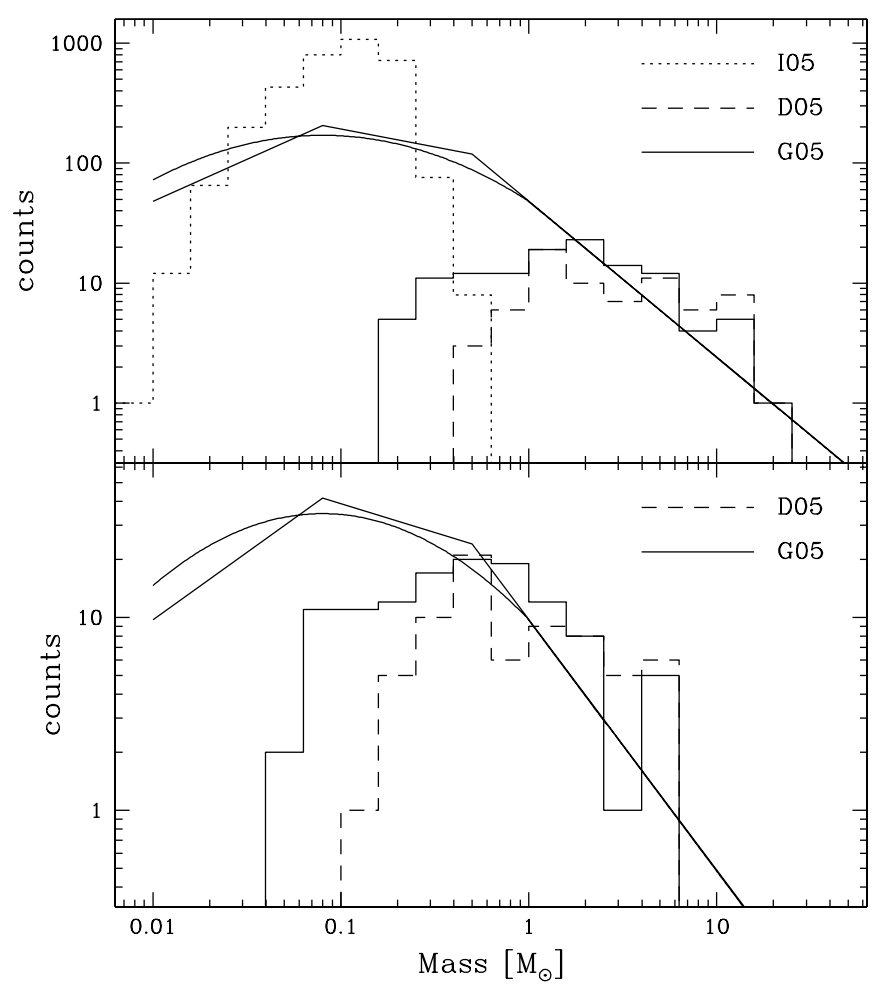

Figure 10. Top panel: mass histograms for all simulations with $T_{\min }=5 \mathrm{~K}$. Mass function is shown at the final time $\left(2.5 t_{\mathrm{ff}}\right.$ for I05 and D05, 2.4t for G05). The empirical IMF from Chabrier (2003) and Kroupa (2002) are shown, normalized to a maximum mass of $\sim 20 M_{\odot}$. Bottom panel: mass histograms for runs D05 and G05, and efficiency of 30\%.

of sinks in the data from our simulations down by a factor of 0.30 . We then recalculate the shape of the empirical stellar IMF for the new maximum masses and total number of stars. We show the results in the bottom panel of Figure 10. We plot the empirical IMF normalized to the new maximum mass. Our results exhibit better agreement with a stellar mass function with a $30 \%$ efficiency factor. Since we have only decreased the masses, the high-mass slope in our new simulation data remains the same and agrees with the empirical IMF. In addition, the peak in the IMF shows better agreement. At $0.1 M_{\odot}$, our new mass function (for the complete energetics algorithm) is only missing a factor of 2-3 objects compared to the empirical IMF, which is significantly less than the factor of $\sim 20$ seen in Figure 10. However, the adjusted mean and median masses are still higher than in the empirical IMFs (Table 6). Since we assumed that all the mass of the sink went into the star in order to calculate luminosities, it is not self-consistent to apply the efficiency factor after the calculation. We do it only to suggest avenues for future exploration. There are questions about the connection between the core mass function and the stellar mass function (e.g., Clark et al. 2007; Smith et al. 2009). Other effects may decrease the mass of the final stars, including disk fragmentation to form binary or multiple stars and brown dwarfs (e.g., Stamatellos et al. 2011).

\section{SUMMARY AND CONCLUSION}

The motivation for this work was to explore the effect of dust-gas energetics in a clustered star formation simulation. We have presented the results of two new simulations (G05 and G10), which include the complete energetics algorithm discussed in UED09. The properties of the models, including two previous ones (I05 and D05), are summarized in Table 1, and we compare them here.

As in D05 and I05, sink particles, representing protostars, form along filaments and especially at intersections of filaments. The temperature of a simulation which included gas cooling was on average lower than in a simulation with $T_{\mathrm{K}}=T_{\text {dust }}$, as expected. With a lower average temperature, fragmentation was more prevalent and more objects were able to form. The average density profile parameters surrounding a sink were similar among the four simulations and agreed with observations of low-mass Class 0 sources. However, the infall speeds were significantly supersonic, and mass accretion rates were high, both in contrast to observations of low-mass protostars. Infall speeds and mass accretion rates for high-mass protostars and protostars in clustered environments are poorly constrained. Infall speeds and mass accretion rates are somewhat smaller for G05 and G10 compared to D05.

We added a calculation of the ratio of far-infrared luminosity ( $L_{\mathrm{FIR}}$ ) over the SFR to test the use of $L_{\mathrm{FIR}}$ as an SFR tracer in very young regions of clustered star formation. We found that $L_{\mathrm{FIR}} / \mathrm{SFR}$ increases rapidly during the simulation, but that it is significantly lower (factor of 10) than the ratio used to measure extragalactic SFR at the end of the simulations (around 0.7 Myr). Measurements of SFR for very young clusters (ages $<1 \mathrm{Myr}$ ) using $L_{\mathrm{FIR}}$ are very likely underestimated.

We computed the mass evolution of protostars during the simulation and compared the mass function at the end of each simulation. In our previous work, UME10, we found that a non-isothermal, stellar-source-dependent energetics algorithm radically reduced the number of young stars that were formed and formed more massive stars, compared to simulations with isothermal gas. However, the simulations in UME10 overproduced high-mass objects and missed a large fraction of low-mass objects. We predicted in UME10 that including a more realistic calculation of the gas temperature might address this problem. In this work, we included the complete dust-gas energetics algorithm. This change increased the number of intermediate-mass objects, but the deficiency of low-mass objects persists.

The two main differences between D05 versus G05 and G10 were the temperature distribution and the mass function, which are related to each other. In a lower temperature environment with more sink particles forming, there was less material available to be accreted and therefore a smaller fraction of massive objects were formed. This affected the mass function and led to a slight decrease in the number of high-mass objects and an increase in the amount of low-mass objects when compared to the simulations with dust heating only in UME10. We found very little difference in the mass function between G05 and G10, indicating that the initial temperature is not very important; feedback from the first protostars rapidly erases the effects of initial temperature.

We performed a thought experiment in which we tried to explain the discrepancy between our mass function and the empirical IMF. In our simulation, we assumed that all the mass in a sink particle winds up in a single star. However, studies of nearby clouds (e.g., Alves et al. 2007; Enoch et al. 2008) show that about $70 \%$ of the core mass does not wind up in the star, probably because it is removed by stellar winds and the resulting molecular outflow (e.g., Dunham et al. 2010). If we multiply our sink masses by 0.3 , we get better agreement with the IMF. Although we have shown that including dust-gas energetics is essential, other effects (e.g., magnetic fields, turbulence, outflows, etc.) will need to be included for a full understanding. 
In a promising development, Hansen et al. (2012) found that including outflows along with radiative feedback reduced the mass accretion rates and protostellar masses, hence luminosities, allowing more fragmentation and better reproducing the IMF. More recently, Krumholz et al. (2012) have found better agreement with the IMF when turbulence, outflows, and radiative transport are included, although their IMFs are still somewhat top-heavy.

All calculations were performed at the Laboratoire d'astrophysique numérique, Université Laval. We are pleased to acknowledge the support of NASA Grants NAG5-10826 and NAG5-13271. We also thank the Canada Research Chair program (H.M.), NSERC (H.M.), NSF Grants AST-0607793 and AST-1109116 (N.E.), and the NASA GSRP Fellowship Program (A.U.) for providing support for this work. Part of A. U.'s contribution to the research described in this publication was carried out at the Jet Propulsion Laboratory, California Institute of Technology, under a contract with the National Aeronautics and Space Administration.

\section{REFERENCES}

Alcala, J. M., Krautter, J., Covino, E., et al. 1997, A\&A, 319, 184

Alves, J., Lombardi, M., \& Lada, C. J. 2007, A\&A, 462, L17

Bastian, N., Covey, K. R., \& Meyer, M. R. 2010, ARA\&A, 48, 339

Bate, M. R. 2005, MNRAS, 363, 363

Bate, M. R. 2009, MNRAS, 392, 1363

Bate, M. R., Bonnell, I. A., \& Bromm, V. 2003, MNRAS, 339, 577

Bonnell, I. A., Clarke, C. J., \& Bate, M. R. 2006, MNRAS, 368, 1296

Bressert, E., Bastian, N., Gutermuth, R., et al. 2010, MNRAS, 409, L54

Briceño, C., Luhman, K. L., Hartmann, L., Stauffer, J. R., \& Kirkpatrick, J. D. 2002, ApJ, 580, 317

Bromm, V., Coppi, P. S., \& Larson, R. B. 2002, ApJ, 564, 23

Chabrier, G. 2003, PASP, 115, 763

Clark, P. C., Glover, S. C. O., \& Klessen, R. S. 2008, ApJ, 672, 757

Clark, P. C., Klessen, R. S., \& Bonnell, I. A. 2007, MNRAS, 379, 57

Comerón, F., Spezzi, L., \& López Martí, B. 2009, A\&A, 500, 1045

Dale, J. E., Bonnell, I. A., Clarke, C. J., \& Bate, M. R. 2005, MNRAS, 358, 291

Dunham, M. M., Evans, N. J., Terebey, S., Dullemond, C. P., \& Young, C. H. 2010, ApJ, 710, 470

Enoch, M. L., Evans, N. J., II, Sargent, A. I., et al. 2008, ApJ, 684, 1240

Gao, Y., \& Solomon, P. M. 2004, ApJ, 606, 271

Goldsmith, P. F. 2001, ApJ, 557, 736

Gutermuth, R. A., Megeath, S. T., Myers, P. C., et al. 2009, ApJS, 184, 18

Hansen, C. E., Klein, R. I., McKee, C. F., \& Fisher, R. T. 2012, ApJ, 747, 22
Heitsch, F., Hartmann, L. W., Slyz, A. D., Devriendt, J. E. G., \& Burkert, A. 2008, ApJ, 674, 316

Hennemann, M., Motte, F., Schneider, N., et al. 2012, A\&A, 543, L3

Hillenbrand, L. A. 1997, AJ, 113, 1733

Hollenbach, D., \& McKee, C. F. 1989, ApJ, 342, 306

Jappsen, A.-K., Klessen, R. S., Larson, R. B., Li, Y., \& Mac Low, M.-M. 2005, A\&A, 435, 611

Kennicutt, R. C., Jr. 1998, ARA\&A, 36, 189

Kitsionas, S., \& Whitworth, A. P. 2002, MNRAS, 330, 129

Klessen, R. S., Burkert, A., \& Bate, M. R. 1998, ApJ, 501, L205

Kroupa, P. 2002, Science, 295, 82

Krumholz, M. R., Cunningham, A. J., Klein, R. I., \& McKee, C. F. 2010, ApJ, 713,1120

Krumholz, M. R., Klein, R. I., \& McKee, C. F. 2007, ApJ, 656, 959

Krumholz, M. R., Klein, R. I., \& McKee, C. F. 2012, ApJ, 754, 71

Krumholz, M. R., \& McKee, C. F. 2005, ApJ, 630, 250

Krumholz, M. R., \& Tan, J. C. 2007, ApJ, 654, 304

Lada, C. J., \& Lada, E. A. 2003, ARA\&A, 41, 57

Larson, R. B. 2005, MNRAS, 359, 211

Li, Y., Klessen, R. S., \& Mac Low, M.-M. 2003, ApJ, 592, 975

Martel, H., Evans, N. J., II, \& Shapiro, P. R. 2006, ApJS, 163, 122 (MES06)

McKee, C. F., \& Ostriker, E. C. 2007, ARA\&A, 45, 565

Miller, G. E., \& Scalo, J. M. 1979, ApJS, 41, 513

Monaghan, J. J. 1992, ARA\&A, 30, 543

Nenkova, M., Ivezić, Ž., \& Elitzur, M. 2000, in ASP Conf. Ser. 196, Thermal Emission Spectroscopy and Analysis of Dust, Disks, and Regoliths, ed. M. L. Sitko, A. L. Sprague, \& D. K. Lynch (San Francisco, CA: ASP), 77

Offner, S. S. R., Klein, R. I., McKee, C. F., \& Krumholz, M. R. 2009, ApJ, 703, 131

Ossenkopf, V., \& Henning, T. 1994, A\&A, 291, 943

Salpeter, E. E. 1955, ApJ, 121, 161

Scalo, J. M. 1986, Fundam. Cosm. Phys., 11, 1

Shirley, Y. L., Evans, N. J., II, \& Rawlings, J. M. C. 2002, ApJ, 575, 337

Smith, R. J., Longmore, S., \& Bonnell, I. 2009, MNRAS, 400, 1775

Stamatellos, D., Maury, A., Whitworth, A., \& André, P. 2011, MNRAS, 413, 1787

Urban, A., Evans, N. J., \& Doty, S. D. 2009, ApJ, 698, 1341 (UED09)

Urban, A., Martel, H., \& Evans, N. J. 2010, ApJ, 710, 1343 (UME10)

van der Tak, F. F. S., \& van Dishoeck, E. F. 2000, A\&A, 358, L79

Vutisalchavakul, N., \& Evans, N. J., II 2012, ApJ, submitted

Williams, J. P., Blitz, L., \& McKee, C. F. 2000, in Protostars and Planets IV, ed. V. Mannings, A. P. Boss, \& S. S. Russell (Tucson, AZ: Univ. Arizona Press), 97

Wuchterl, G., \& Tscharnuter, W. M. 2003, A\&A, 398, 1081

Wu, J., Evans, N. J., II, Gao, Y., et al. 2005, ApJ, 635, L173

Wu, J., Evans, N. J., II, Shirley, Y. L., \& Knez, C. 2010, ApJS, 188, 313

Young, C. H., \& Evans, N. J., II 2005, ApJ, 627, 293

Young, C. H., Shirley, Y. L., Evans, N. J., II, \& Rawlings, J. M. C. 2003, ApJS, 145,111

Young, K. E., Lee, J.-E., Evans, N. J., II, Goldsmith, P. F., \& Doty, S. D. 2004, ApJ, 614, 252 